\title{
Highlights of GeV gamma-ray astronomy
}

\author{
D. J. Thompson \\ NASA Goddard Space Flight Center, Greenbelt, MD USA \\ On Behalf of the Fermi Large Area Telescope Collaboration
}

Received: 18 October 2010 - Accepted: 24 November 2010 - Published: 20 December 2010

\begin{abstract}
Because high-energy gamma rays are primarily produced by high-energy particle interactions, the gammaray survey of the sky by the Fermi Gamma-ray Space Telescope offers a view of sites of cosmic ray production and interactions. Gamma-ray bursts, pulsars, pulsar wind nebulae, binary sources, and Active Galactic Nuclei are all phenomena that reveal particle acceleration through their gamma-ray emission. Diffuse Galactic gamma radiation, Solar System gamma-ray sources, and energetic radiation from supernova remnants are likely tracers of high-energy particle interactions with matter and photon fields. This paper will present a broad overview of the constantly changing sky seen with the Large Area Telescope (LAT) on the Fermi spacecraft.
\end{abstract}

\section{Introduction}

High-energy gamma rays are primarily produced by interactions of energetic particles. Typical processes are inelastic nuclear collisions (pion production), inverse Compton scattering, curvature radiation, and synchrotron radiation. In addition, the Universe is mainly transparent to gamma rays with energies less than $20 \mathrm{GeV}$; therefore they can probe distant or obscured regions. These features give gamma-ray observations the potential to image regions of cosmic-ray acceleration and interaction.

The Fermi Gamma-ray Space Telescope was launched on 11 June 2008. Since August 2008, the Fermi Large Area Telescope (LAT, Atwood et al., 2009), the principal instrument on the satellite, has been surveying the sky at energies from $20 \mathrm{MeV}$ to more than $300 \mathrm{GeV}$, taking advantage of its huge field of view $(2.4 \mathrm{sr})$ and the scanning mode of operation of the satellite to view the entire sky every three hours.

Correspondence to: D. J. Thompson (david.j.thompson@nasa.gov)
The present paper reviews some highlights of results from the Fermi LAT, with emphasis on connections to leptonic and hadronic cosmic-ray studies.

\section{Overview of the gamma-ray sky}

The full gamma-ray sky as seen by the LAT is dominated by diffuse emission from the bright plane of our Galaxy. Sources are seen as excesses above the diffuse backgrounds found in all parts of the sky. The first Fermi LAT catalog (Abdo et al., 2010a) contains 1451 sources. At least two populations of sources are seen: an isotropic component, likely representing extragalactic sources, and a clustering of sources along the Galactic plane. For this catalog, the LAT team has used the term "associations" for sources that have positional agreements with known objects, reserving the term "identifications" for sources with time variability, spectral, or spatial features that provide stronger evidence about the nature of the source.

\section{Gamma-ray bursts (GRB)}

The brightest and most distant gamma-ray sources do not appear in the LAT catalog. These are gamma-ray bursts, intense flashes lasting from a fraction of a second to many minutes. GRB are thought to result from particularly powerful supernovae or from the mergers of neutron stars and black holes. GRB results combine data from the LAT with those from the other Fermi instrument, the $8 \mathrm{keV}$ to $40 \mathrm{MeV}$ Gamma-ray Burst Monitor (GBM, Meegan et al., 2009), to cover more than 6 orders of magnitude in photon energies. Some of the Fermi results on GRB include:

- Both long $(>2 \mathrm{~s})$ and short $(<2 \mathrm{~s})$ bursts have been seen by both instruments.

Published by Copernicus Publications on behalf of the Arbeitsgemeinschaft Extraterrestrische Forschung e.V. 
- Some bursts show high-energy emission afterglows, lasting for many minutes after the prompt emission (e.g. DePasquale et al., 2010).

- Fermi observations of some bursts, under reasonable assumptions, put lower limits on bulk Lorentz factors to be greater than 1000 (e.g. Ackermann et al., 2010c).

- Some bursts have an extra spectral component compared to the standard Band model (e.g. Abdo et al., 2009e).

- GRB seen with the LAT have placed constraints on models of quantum gravity that predict Lorentz Invariance violation (Abdo et al., 2009a).

\section{Active Galactic Nuclei (AGN)}

More than half the bright sources seen with the Fermi LAT are associated with Active Galactic Nuclei (AGN), as indicated in the first LAT catalog (Abdo et al., 2010a) and first LAT AGN catalog (Abdo et al., 2010d). In standard models, the energy that powers the nucleus of an active galaxy comes from material falling toward a supermassive black hole. In a process not fully understood, some of this energy ends up in a jet of high-energy particles that travel away from the nucleus at relativistic speeds. The Fermi LAT sees primarily blazars, a subset of radio-loud AGN for which the jet is pointed toward Earth.

Observations of correlated multiwavelength variability, in addition to confirming identifications for AGN gamma-ray sources, also help in modeling these jets. An example is PKS $1502+106$, which exhibited a strong flare early in the mission (Abdo et al., 2010e). In this case, the gamma-ray and Xray flare seem correlated, followed by a delayed flare seen in optical and ultraviolet light and a still later peak in the radio. This last feature is one noted in earlier blazar flares by Valtaoja and Teräsranta (1996). In this case, the pattern suggests particle acceleration and interaction near the outer part of the Broad Line Region of the quasar.

The Spectral Energy Distribution (SED) of this blazar is complex, requiring multiple components that vary with time. Not all AGN flares show the same pattern of temporalspectral variability, leading to a key result for Fermi and multiwavelength studies: in most cases, simple models for blazar jets are inadequate.

Spatially resolving AGN at gamma-ray energies generally not feasible. An exception is Centaurus A, a nearby radio galaxy. The giant radio lobes of Cen A, which span about $10^{\circ}$ on the sky, are visible in the LAT data (Abdo et al., 2010f). Over half of the total observed LAT flux $>100 \mathrm{MeV}$ appears in the lobes. Such emission requires $0.1-1 \mathrm{TeV}$ electrons in these lobes, either accelerated in the lobes or efficiently transported from the core of the galaxy.

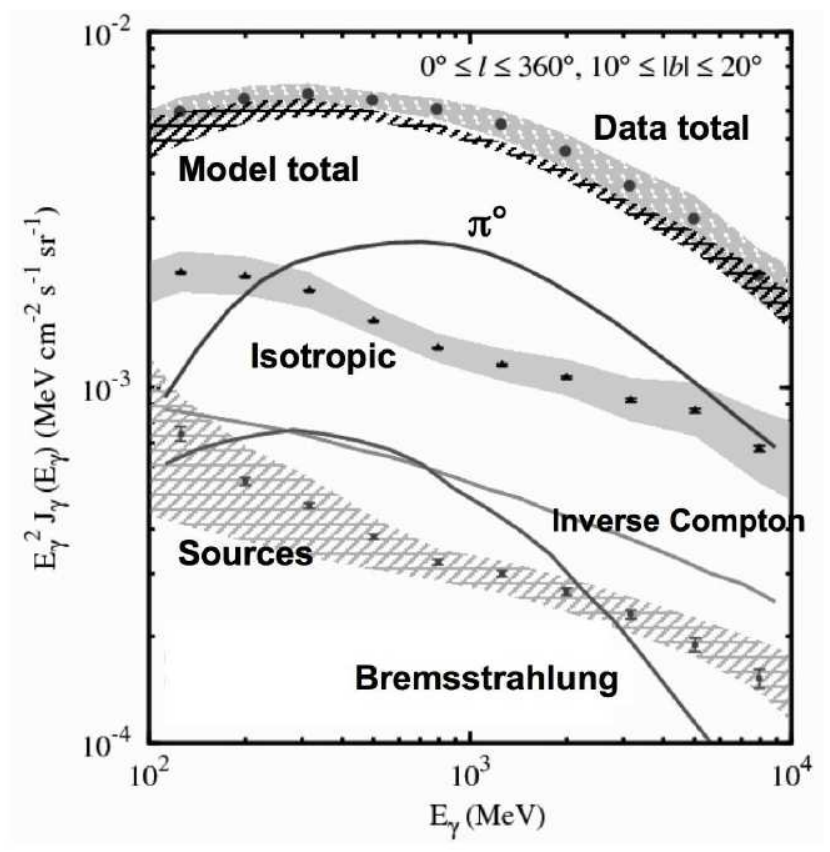

Fig. 1. Energy spectrum of diffuse gamma radiation at intermediate Galactic latitudes. Overlaid are SEDs for the component processes of the diffuse emission. The dominant component, shown by the heavier black line above the gray band, is decay of $\pi^{0}$ mesons (Abdo et al., 2009f).

\section{Galaxies dominated by cosmic-ray interactions}

Some galaxies not classed as AGN are also seen as gammaray sources. Examples are normal galaxies, including the Milky Way and the Large Magellanic Cloud (Abdo et al., $2010 \mathrm{~g}$ ), and starburst galaxies, such as M82 and NGC 253 (Abdo et al., 2010h). GeV gamma rays in these galaxies come primarily from the interactions of cosmic ray hadrons and electrons with interstellar matter and photon fields.

The gamma-ray luminosities of these normal and starburst galaxies show an approximately linear relationship with the product of the supernova rate and the total mass of gas in the galaxies (Abdo et al., 2010h). Although this analysis is highly simplified, the trend supports the long-held expectation that supernovae are principal accelerators of cosmic rays (at least for hadrons).

\section{Diffuse gamma radiation in the Milky Way}

Although the diffuse Galactic gamma radiation is brightest along the plane of the Milky Way, it is seen at all Galactic latitudes. This radiation is largely produced by cosmic-ray interactions with the interstellar gas and photon fields through the processes of inelastic nucleon scattering, bremsstrahlung, and inverse Compton scattering. Figure 1 shows the spectrum of this radiation in part of the sky, along with modeled components (Abdo et al., 2009f). The largest contributor to 
this radiation is nucleon-nucleon collisions with subsequent decay of $\pi^{0}$ mesons into gamma rays in the Fermi LAT energy range. The LAT measurements do not confirm the unexpected excess in the few $\mathrm{GeV}$ energy range measured by EGRET on the Compton Gamma Ray Observatory (CGRO) compared to models.

Despite being known from the beginning of gamma-ray astrophysics, the diffuse Galactic radiation has proven challenging to model in detail. Large-scale features are modeled reasonably well by physical models that use measured gas and photon distributions together with cosmicray propagation calculations. The current standard for such work is GALPROP. A recent summary is given by Strong, Moskalenko, and Ptuskin (2007). Fermi LAT observations, however, imply some features about the cosmic-ray distribution that differ from the standard model: the cosmic-ray gradient in the outer Galaxy is shallower than expected (Abdo et al., 2010i) and the cosmic-ray halo scale height may be larger than previously assumed.

A GALPROP model for the diffuse gamma radiation was used in the first analysis of LAT sources, the Bright Source List (Abdo et al., 2009g). By the time of the first full LAT catalog, however, the LAT team found that a diffuse model optimized for point source analysis was required (Abdo et al., 2010a). Studies of the diffuse radiation, including the complex Galactic Center region, are ongoing.

\section{Galactic gamma-ray sources}

The cosmic-ray particles that permeate the local interstellar medium are likely to be produced by the same phenomena that are seen as high-energy gamma-ray sources. The Fermi LAT results have substantially expanded our knowledge of such objects.

\subsection{Pulsars, pulsar wind nebulae, and binary systems}

Gamma-ray pulsars were the first class of sources identified at these energies. The first Fermi LAT pulsar catalog (Abdo et al., 2010b) includes 46 pulsars, and more continue to be identified. In addition to the young, radio-bright pulsars that were well known from the CGRO era, LAT observations have added three new aspects to pulsar studies:

1. A number of millisecond pulsars are visible in the LAT data (Abdo et al., 2009b). These old, recycled pulsars that have passed through a stage of being X-ray binaries are similar in their gamma-ray properties to the younger pulsars despite having much weaker surface magnetic fields and much faster rotation speeds.

2. A new population of gamma-ray-selected pulsars was discovered in blind searches of gamma-ray arrival times from some bright unidentified LAT sources (Abdo et al., $2009 \mathrm{c}$ ), suggesting that the gamma-ray beams from at least some of these pulsars may be broader than the radio or X-ray beam.

3. In a multiwavelength effort, radio astronomers have discovered a number of new millisecond pulsars in error boxes of unidentified LAT sources, often assisted by source locations derived from X-ray observations.

In terms of cosmic rays, these pulsars may be of greatest interest as possible sources of high-energy electrons. The LAT results on cosmic-ray electrons (Abdo et al., 2009h) suggest a local source of electrons in the energy range just below $1 \mathrm{TeV}$, and pulsars are candidates to provide those.

Much of the energy losses of pulsars as they slow down goes into the production of a pulsar wind, and the termination shock of this wind can accelerate particles (primarily electrons, although proton acceleration cannot be ruled out) and produce a Pulsar Wind Nebula (PWN). The interacting PWN particles can then produce gamma rays. The LAT has seen several PWNe, including the Crab (Abdo et al., 2010j), where the LAT results connect to the observations from $\mathrm{TeV}$ Atmospheric Cherenkov telescopes, completing the picture of synchrotron plus inverse Compton scattering across the spectrum, and the Vela X PWN (Abdo et al., 2010k), where the $\mathrm{GeV}$ and $\mathrm{TeV}$ emissions appear to originate from different electron populations, indicating both recent and past episodes of particle acceleration.

Another class of gamma-ray sources seen by the LAT includes some High-Mass X-ray Binary (HMXB) systems, where the variation in the gamma-ray flux originates from the orbital motion of the system. One such system is LS5039, which has a neutron star or black hole in a 3.9-day orbit around a massive star. The $\mathrm{GeV}$ gamma radiation clearly displays the orbital modulation (Abdo et al., 2009i), although the modulation is out of phase with the TeV emission seen from the same system. These HMXB systems are not well enough understood to know whether leptonic or hadronic cosmic-ray production and interactions dominate the gamma-ray flux.

\subsection{Supernova remnants (SNR)}

Supernovae and supernova remnants have long been thought to be sites of cosmic-ray particle acceleration (e.g. Ginzburg and Syrovatskii, 1964), and the collisions of these newly accelerated particles with the surrounding interstellar medium should then produce gamma rays by the same processes that produce the diffuse Galactic gamma radiation. The sensitivity and resolution of the Fermi LAT has now provided strong evidence in several cases for just such processes taking place.

One example is Supernova Remnant W44, which is spatially resolved by the LAT using $2-10 \mathrm{GeV}$ gamma rays that convert in the front part of the detector with the best angular resolution (Abdo et al., 20101). The gamma-ray image resembles the shell structure seen with the Spitzer Infrared Array Camera (Reach et al., 2006), thought to represent dense 


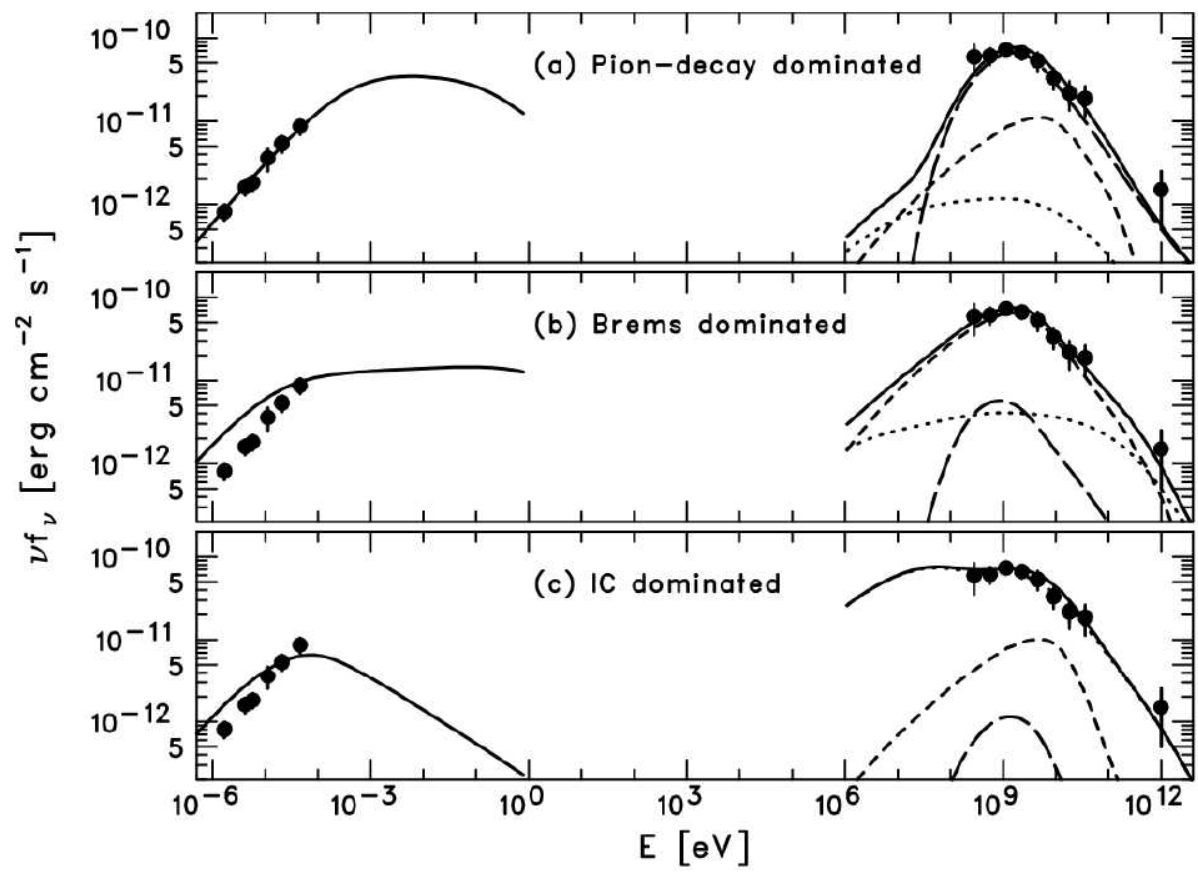

Fig. 2. Spectral Energy Distribution of radio and gamma-ray emission from SNR 51C (Abdo et al., 2009j). The radio emission (e.g. Moon and Koo, 1994) is explained by synchrotron radiation, while the gammaray emission is modeled by different combinations of $\pi^{0}$-decay (longdashed curve), bremsstrahlung (dashed curve), and Inverse Compton scattering (dotted curve). The sum of the three components is shown as a solid curve. clouds of shocked molecular hydrogen. These clouds then form the targets for the cosmic rays accelerated in the SNR. The gamma-ray spectrum is consistent with $\pi^{0}$-decay, although it is possible to construct models in which the emission has a leptonic origin.

Another example is Supernova Remnant W51C, which again is spatially resolved by the LAT (Abdo et al., 2009j). This SNR also contains shocked molecular clouds that could be targets for cosmic-ray interactions. Figure 2 shows the radio and gamma-ray spectrum as measured and modeled three different ways. Although the hadronic and leptonic models can all explain the gamma-ray spectrum, the model parameters required by the gamma-ray observations predict different synchrotron radio spectra, and these appear most consistent with the $\pi^{0}$-decay-dominated gamma-ray calculation.

With the number of SNR seen by the Fermi LAT growing, and with the multiwavelength studies supporting an origin of the gamma rays in hadronic processes, the evidence is mounting for the long-conjectured hypothesis that SNR are the source of the bulk of Galactic cosmic rays. The sample is still small enough, however, that declaring the quest over seems premature.

\subsection{A gamma-ray nova}

In March 2010, the LAT data revealed a new, flaring gammaray source in the Cygnus region of the sky (Abdo et al., $2010 \mathrm{~m}$ ), coincident in time and spatial coordinates with a flare seen from the symbiotic binary system V407 Cygni (Nishiyama and Kabashima, 2010). The classical nova outburst from this red giant/white dwarf system was also seen in radio and X-rays, confirming its nonthermal nature. Particles are accelerated in the expanding shell of the nova, interacting with the wind from the red giant and with the surrounding medium to produce the observed gamma radiation. As seen in Fig. 3, the gamma-ray energy spectrum can be modeled as either $\pi^{0}$-decay or leptonic processes (Abdo et al., $2010 \mathrm{~m}$ ). Although novae are far less powerful than supernovae, they are far more numerous. The LAT detection suggests that yet another class of objects can accelerate particles to cosmic-ray energies.

\subsection{Solar system gamma-ray sources}

The Moon and the quiet Sun are both passive sources of high-energy gamma rays, resulting from cosmic-ray interactions. For the Moon, nucleonic interactions with the lunar surface produce cascades that include gamma rays (Giglietto and Fermi LAT Collaboration, 2009). The same processes occur on the Sun, although the cosmic-ray flux is depleted by the solar magnetic field. An additional, spatially extended solar gamma-ray component originates from inverse Compton scattering of cosmic-ray electrons with the solar photon field (Orlando, Giglietto and Fermi LAT Collaboration, 2009). The gamma radiation from both the Moon and the Sun are affected by the solar cycle modulation of cosmic radiation. 


\section{The future of $\mathrm{GeV}$ gamma-ray observations with Fermi}

The Fermi Gamma-ray Space Telescope and both its scientific instruments are working well at the end of more than two years of operation. The mission is planned for five years, and the fact that there are no consumables means that the full duration of the mission could be much longer. For a variety of reasons, the prospects for continued scientific advances with Fermi are excellent. Some of these are:

- The gamma-ray sky is changing every day, so there is always something new to learn about the extreme Universe. More than 100 Astronomer's Telegrams have been published based on Fermi observations of variability in sources, a rate of nearly one per week. The dynamic nature of the gamma-ray sky offers continual opportunities for new studies.

- Even for persistent sources, the accumulation of photons adds significantly to the scientific return. Better source locations and improved mapping emerge as more of the highest-energy LAT gamma rays are detected, because the point-spread function improves with increasing energy. Increased counting statistics also enable deeper searches for fainter sources.

- The LAT gamma-ray data are public, released as soon as the basic processing is done. The LAT team encourages use of the data, recognizing that scientists outside the LAT team bring added insight to gamma-ray astrophysics. The Fermi Science Support Center, at http://fermi.gsfc.nasa.gov/ssc/ is the access center for these data, along with analysis software, documentation, and diffuse models needed for analysis of the data. The LAT team also welcomes cooperative efforts in all areas of gamma-ray astrophysics.

- Improvements in the LAT diffuse model and the analysis procedures are constantly being tested and will be made public as soon as the LAT team has confidence in them.

- A broad expanse of discovery space remains for studies with the Fermi Gamma-ray Space Telescope. Beyond pulsars, blazars, radio galaxies, X-ray binaries, SNR, starburst galaxies, stellar novae, and gamma-ray bursts, other sources remain mysteries. Nearly $40 \%$ of the sources in the First LAT Catalog do not seem to have obvious counterparts at other wavelengths. Multiwavelength/multimessenger studies will be critical for learning the nature of such sources. Topics such as the search for gamma-ray evidence of dark matter and the limits on the resolution of the nature of the diffuse extragalactic radiation remain open questions.

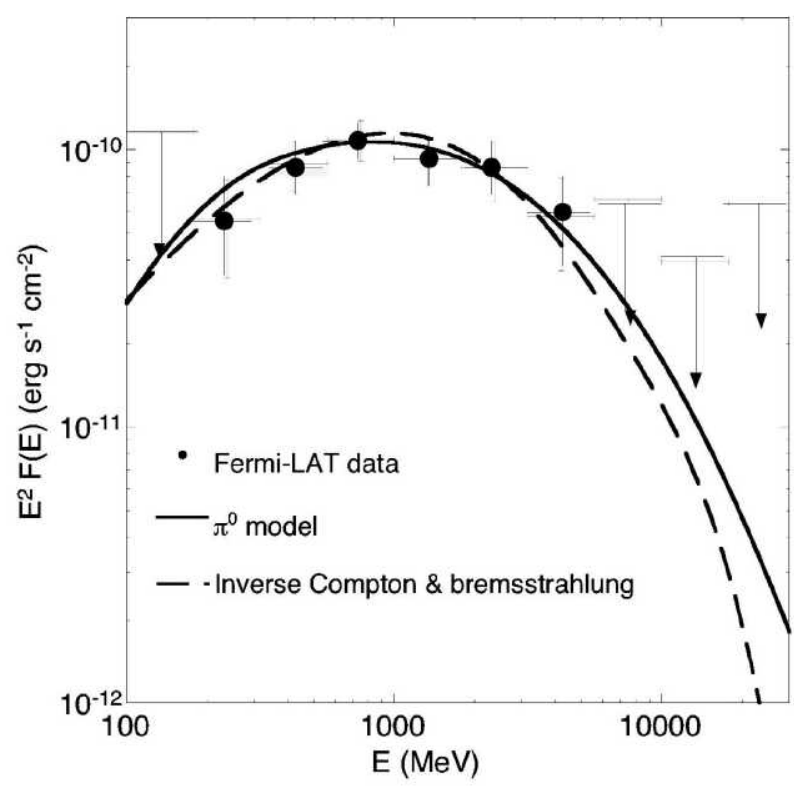

Fig. 3. Gamma-ray energy spectrum of V407 Cygni during the flare (Abdo et al., 2010m).

\section{Conclusions}

Gamma rays seen with the Fermi Gamma-ray Space Telescope are revealing sites of particle acceleration and interaction, ranging from distant Gamma-ray Bursts and Active Galactic Nuclei to sources nearby in our own Galaxy and even within the Solar System. Many of these gamma-ray sources have direct implications for cosmic-ray physics.

The Fermi LAT results support, but have yet to prove definitively, the idea that supernovae are a primary source of cosmic rays. Pulsars and binary systems contribute to the cosmic-ray electron population. Novae are also potential cosmic-ray accelerators.

The Fermi mission is planned to continue at least three more years, and many discovery opportunities remain. All the Fermi gamma-ray data are public. The future of cooperative gamma-ray and cosmic-ray studies is bright.

Acknowledgements. The Fermi LAT Collaboration acknowledges generous ongoing support from a number of agencies and institutes that have supported both the development and the operation of the LAT as well as scientific data analysis. These include the National Aeronautics and Space Administration and the Department of Energy in the United States, the Commissariat à l'Energie Atomique and the Centre National de la Recherche Scientifique / Institut National de Physique Nucléaire et de Physique des Particules in France, the Agenzia Spaziale Italiana and the Istituto Nazionale di Fisica Nucleare in Italy, the Ministry of Education, Culture, Sports, Science and Technology (MEXT), High Energy Accelerator Research Organization (KEK) and Japan Aerospace Exploration Agency (JAXA) in Japan, and the K. A. Wallenberg Foundation, the Swedish Research Council and the Swedish National Space Board in Sweden. 
Additional support for science analysis during the operations phase is gratefully acknowledged from the Istituto Nazionale di Astrofisica in Italy and the Centre National d'Études Spatiales in France.

Edited by: J. Poutanen

Reviewed by: two anonymous referees

\section{References}

Abdo, A.A., Ackermann, M., Ajello, M. et al.: A limit on the variation of the speed of light arising from quantum gravity effects, Nature, 462, 331-334, 2009a.

Abdo, A.A., Ackermann, M., Ajello, M. et al: A Population of Gamma-Ray Millisecond Pulsars Seen with the Fermi Large Area Telescope, Science, 325, 848-852, 2009b.

Abdo, A.A., Ackermann, M., Ajello, M. et al.: Detection of 16 Gamma-Ray Pulsars Through Blind Frequency Searches Using the Fermi LAT, Science, 325, 840-844, 2009c.

Abdo, A.A., Ackermann, M., Ajello, M. et al.: Fermi Observations of GRB 090902B: A Distinct Spectral Component in the Prompt and Delayed Emission, Astroph. J., 706, L138-L144, 2009e .

Abdo, A.A., Ackermann, M., Ajello, M. et al.: Fermi Large Area Telescope Measurements of the Diffuse Gamma-Ray Emission at Intermediate Galactic Latitudes, Phys. Rev. Lett., 103, 251 101105, 2009f.

Abdo, A.A., Ackermann, M., Ajello, M. et al.: Fermi/Large Area Telescope Bright Gamma-Ray Source List, Astroph. J. Supp., $183,46-66,2009 \mathrm{~g}$.

Abdo, A.A., Ackermann, M., Ajello, M. et al.: Measurement of the Cosmic Ray $\mathrm{e}^{+}+\mathrm{e}^{-}$Spectrum from $20 \mathrm{GeV}$ to $1 \mathrm{TeV}$ with the Fermi Large Area Telescope, Phys. Rev. Lett., 102, 181 101-105, $2009 \mathrm{~h}$

Abdo, A.A., Ackermann, M., Ajello, M. et al.: Fermi/LAT observations of LS 5039 , Astroph. J., 706, L56-L61, 2009 i.

Abdo, A.A., Ackermann, M., Ajello, M. et al.: Fermi LAT Discovery of Extended Gamma-Ray Emission in the Direction of Supernova Remnant W51C, Astroph. J., 706, L1-L6, 2009j.

Abdo, A.A., Ackermann, M., Ajello, M. et al.: Fermi Large Area Telescope First Source Catalog, Astroph. J. Supp., 188, 405-436, 2010a.

Abdo, A.A., Ackermann, M., Ajello, M. et al.: The First Fermi Large Area Telescope Catalog of Gamma-ray Pulsars, Astroph. J., 187, 460-494, 2010b.

Abdo, A.A., Ackermann, M., Ajello, M. et al.: The First Catalog of Active Galactic Nuclei Detected by the Fermi Large Area Telescope, Astroph. J., 715, 429-457, 2010d.

Abdo, A.A., Ackermann, M., Ajello, M. et al.: PKS 1502+106: A New and Distant Gamma-ray Blazar in Outburst Discovered by the Fermi Large Area Telescope, Astroph. J., 710, 810-827, 2010e.

Abdo, A.A., Ackermann, M., Ajello, M. et al.: Fermi Gamma-Ray Imaging of a Radio Galaxy, Science, 328, 725-730, $2010 \mathrm{f}$.

Abdo, A.A., Ackermann, M., Ajello, M. et al.: Observations of the Large Magellanic Cloud with Fermi, A and A, 512, 7-12, $2010 \mathrm{~g}$.
Abdo, A.A., Ackermann, M., Ajello, M. et al.: Detection of Gamma-Ray Emission from the Starburst Galaxies M82 and NGC 253 with the Large Area Telescope on Fermi, Astroph. J., 709, L152-L157, 2010h.

Abdo, A.A., Ackermann, M., Ajello, M. et al.: Fermi Observations of Cassiopeia and Cepheus: Diffuse Gamma-ray Emission in the Outer Galaxy, Astroph. J., 710, 133-149, $2010 \mathrm{i}$.

Abdo, A.A., Ackermann, M., Ajello, M. et al.: Fermi Large Area Telescope Observations of the Crab Pulsar And Nebula, Astroph. J., 708, 1254-1267, 2010j.

Abdo, A.A., Ackermann, M., Ajello, M. et al.: Fermi Large Area Telescope Observations of the Vela-X Pulsar Wind Nebula, Astroph. J., 713, 146-153, 2010k.

Abdo, A.A., Ackermann, M., Ajello, M. et al.: Gamma-Ray Emission from the Shell of Supernova Remnant W44 Revealed by the Fermi LAT, Science, 327, 1103-1107, 20101.

Abdo, A.A., Ackermann, M., Ajello, M. et al.: Gamma-Ray Emission Concurrent with the Nova in the Symbiotic Binary V407 Cygni, Science, 329, 817-821, 2010m.

Ackermann, M., Asano, K., Atwood, W. B. et al.: Fermi Observations of GRB 090510: A Short-Hard Gamma-ray Burst with an Additional, Hard Power-law Component from $10 \mathrm{keV}$ To GeV Energies, Astroph. J., 716, 1178-1190, 2010c.

Atwood, W. B. Abdo, A.A., Ackermann, M., et al.: The Large Area Telescope on the Fermi Gamma-Ray Space Telescope Mission, Astroph. J., 697, 1071-1102, 2009.

De Pasquale, M., Schady, P., Kuin, N. P. M.; et al.: Swift and Fermi Observations of the Early Afterglow of the Short Gamma-Ray Burst 090510, Astroph. J., 709, L146-L151, 2009.

Giglietto, N. and the Fermi LAT Collaboration: Lunar gamma ray emission seen during the first year by Fermi, 2009 Fermi Symposium, eConf. Proc. C091122, 2009.

Ginzburg, V. L. and Syrovatskii, S. I.: The Origin of Cosmic Rays, New York: Macmillan, 1964.

Meegan, C., Lichti, G., Bhat, P. N., et al.: The Fermi Gamma-ray Burst Monitor, Astroph. J., 702, 791-804, 2009.

Moon, D. S. and Koo, B. C.: Thermal and Non-Thermal Radio Continuum Sources in the W51 Complex, J. Korean Astron. Soc., 27, 81-102, 1994.

Nishiyama, K. and Kabashima, F.: V407 Cygni, Intern. Astron. Union Central Bureau for Astron. Telegrams, reported by $\mathrm{H}$. Maehara, No. 2199, 2010.

Orlando, E., Giglietto, N. and the Fermi LAT Collaboration: FermiLAT Observation of Quiet Solar Emission, 2009 Fermi Symposium, eConf. Proc. C091122, 2009.

Reach, W. T., Rho, J., Tappe, A. et al:: A Spitzer Space Telescope Infrared Survey of Supernova Remnants in the Inner Galaxy, Astron. J., 131, 1479-1500, 2006.

Strong, A. W., Moskalenko, I. V., and Ptuskin, V. S.: Cosmic-Ray Propagation and Interactions in the Galaxy, Ann. Rev. of Nucl. and Part. Science, 57, 285-327, 2007.

Valtaoja, E. and Teräsranta, H.: The relationship between gamma emission and radio flares in AGN, A and A Supp., 120, 491-494, 1996. 